\title{
High-frequency blockade and related phenomena
}

\author{
M.M. Mahmoodian ${ }^{\text {a,b,1 }}{ }^{\text {, M.V. Entin }}{ }^{\text {a }}$ and L.S. Braginsky ${ }^{a}$ \\ ${ }^{a}$ Institute of Semiconductor Physics, Siberian Division of Russian Academy of Sciences, Novosibirsk, 630090, Russia \\ b Novosibirsk State University, Novosibirsk, 630090, Russia
}

\begin{abstract}
We study systems with local vibrating potentials, one-dimensional single and double wells and the tight-binding 1D model with single vibrating site. In general, these systems transmit, or reflect particles inelastically, with absorption or emission of several frequency quanta. Nevertheless, we have found that at some conditions these systems can perfectly and elastically reflect electrons. This high-frequency "blockade" give rise to unique possibility of near-ideal localization of electron with the energy lying on the background of continuous energy spectrum. We discuss different consequences of this statement.
\end{abstract}

Key words: 1D system with vibrating wells, ideal reflectivity, ideal transparency, blockade states, local states, quantum pump

PACS: 73.50.Pz, 73.23.-b, 85.35.Be

\section{Basic formulae.}

We consider 1D systems with potential

$$
\begin{array}{r}
U(x)=\left(u_{1}+v_{1} \sin (\omega t)\right) \delta(x+d)+ \\
\left(u_{2}+v_{2} \sin (\omega t+\varphi)\right) \delta(x-d),
\end{array}
$$

where $t$ is time, $\hbar=m_{e}=1$, the case of single well is specified by $u_{2}=v_{2}=0$ ), and the tight-binding model

$$
i \dot{a}_{m}-\delta_{m, 0}(\widetilde{u}+\widetilde{v} \sin \omega t) a_{0}+\frac{\Delta}{2}\left(a_{m+1}+a_{m-1}\right)=0
$$

Here $a_{m}$ is time-dependent amplitude of the wave function on the $m$-th atom, $\widetilde{u}+\widetilde{v} \sin \omega t$ is the vibrating energy level of the atom $m=0,2 \Delta$ is the width of permitted band, the energy is referred to the band center. The presence of alternating potential leads to change of the dc conductance and appearance of stationary current excited by alternating potential itself (electron pump-

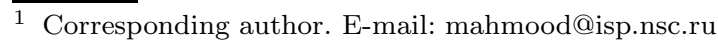

Preprint submitted to Physica E ing $[1,2,3,4,5,6])$. The present paper consider these responses beyond the perturbation theory approximation, that leads to new unusual features.

The electron wave functions outside the wells (barriers) can be searched in the form:

$$
\begin{array}{ll}
\Psi=e^{-i E t}\left\{e^{i p x}+\sum_{n} r_{n} e^{-i n \omega t-i p_{n} x}\right\} & \text { left, } \\
\Psi=e^{-i E t} \sum_{n} e^{-i n \omega t+i p_{n} x} t_{n} & \text { right. }
\end{array}
$$

Here $p_{n}=\sqrt{p^{2}+2 n \omega}, p=\sqrt{2 E}$. In the single-well problem case the transmission coefficients $t_{n}$ obeys

$t_{n}\left(p_{n}+i u\right)+\frac{v}{2}\left(t_{n+1}-t_{n-1}\right)=p_{n} \delta_{n, 0}$.

The Eq. (5) can be found from equations for corresponding double-barrier problem $[4,5]$. At low temperature the conductance $G$, the stationary current $J_{0}$, caused by alternating signal itself, and derivative of $J_{0}$ with respect to the Fermi level $E_{F}$ read:

9 November 2018 


$$
\begin{array}{r}
G=\left.\frac{G_{0}}{2}\left(T^{\rightarrow}+T^{\leftarrow}\right)\right|_{E=E_{F}}, \\
J_{0}=\frac{e}{\pi \hbar} \int_{0}^{E_{F}} d E\left(T^{\rightarrow}-T^{\leftarrow}\right), \\
e \frac{\partial}{\partial E_{F}} J_{0} \equiv S=\left.G_{0}\left(T^{\rightarrow}-T^{\leftarrow}\right)\right|_{E=E_{F}} .
\end{array}
$$

Here arrows mark the direction of motion, $T^{\rightleftarrows}=$ $\sum_{n}\left|t_{n}\right|^{2}, G_{0}=2 e^{2} / h$ is the conductance quantum. The Eq. (6) results from Landauer formula, the Eqs. $(7-8)$ see in $[4,5]$. The stationary current $J_{0}$ exists if the system is asymmetric, $T^{\rightarrow} \neq T^{\leftarrow}$, in particular, it vanishes for single $\delta$-function and the model (2).

Single oscillating $\delta$-function. Conductance and reflection resonances.

Figure 1 presents dependence of conductance on parameters of the potential. Apparently, the conductance

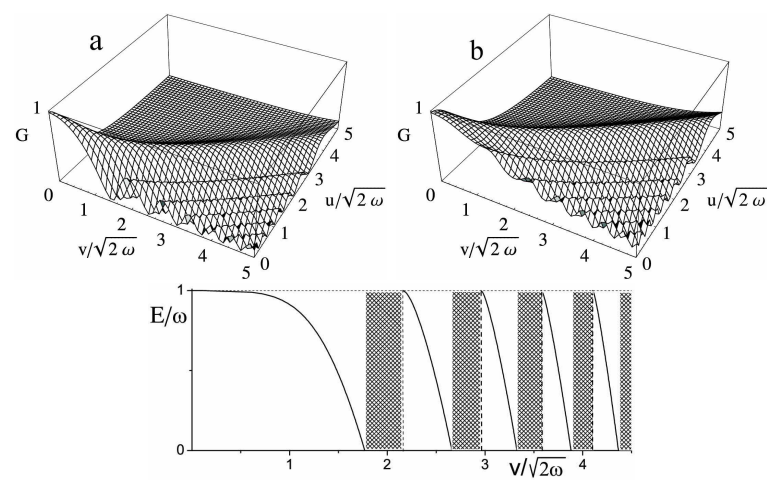

Fig. 1. a,b - Conductance of single vibrating barrier/well in units of $G_{0}$ versus $u$ and $v$. The Fermi energy has values $0.5 \omega(\mathrm{a})$ and $1.5 \omega(\mathrm{b})$. c - Blockade state energy versus value of alternating potential $v$ for $u=0$. Forbidden values of $v$, where blockade states absent are crosshatched.

is oscillating function of the amplitude of vibrations $v$. The conductance vanishes at the lines of the $(v, u)$ plane, if $E_{F}<\omega$; otherwise, if $E_{F}>\omega$, it oscillates remaining positive.

Zeros of the conductance coincides with zeros of the transmittance. It is important that not only transmission, but also inelasticity disappear at the resonance. We call this phenomenon "high-frequency blockade" by analogy with the "Coulomb blockade". Unlike the latter, the "high-frequency blockade" occurs in the absence of interaction between the electrons.

Figure 1c presents dependence of the blockade eigenvalues in the case $u=0$, found by solving the homogeneous system of equations (5) for $n \leq-1$, while for $n \geq 0 t_{n}=0$. The blockade states exist only for $E<$ $\omega$. Only one reflection resonance occurs under small $v$. Note, that zeros of the conductance disappears at some intervals of $v$ (Fig. 1c).

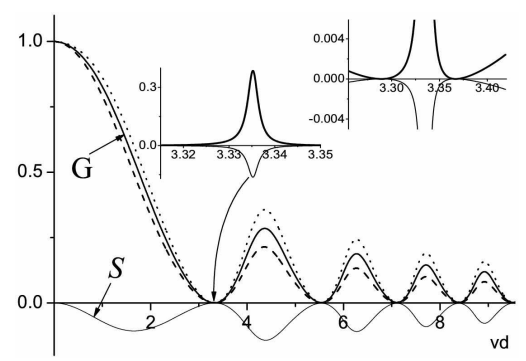

Fig. 2. Conductance (bold) and derivative of photocurrent (thin) in double-well structure versus amplitude of vibrations. The problem parameters: $\omega=5, p_{F}=\pi / 2 d, \varphi=\pi / 2, u_{1}=u_{2}=0, v=v_{1}=v_{2}$. The inserts show magnified vicinity of conductance zeros.

\section{Two oscillating $\delta$-functions.}

This system represents the electron "Fabri-Perrote" interferometer with vibrating mirrors. Due to asymmetry, it can serve as a quantum pump. Figure 2 presents dependence of conductance $G$ and the chemical potential derivative of the photocurrent $S$ on $v=v_{1}=v_{2}$ for $u=0$. The distance between $\delta$-functions is chosen equal to half of the Fermi wavelength. Roughly the dependence resembles that for the case of one $\delta$-function (Fig. 1a). Both values $G$ and $S$ vanish near the same points. However, strong magnification shows that zeros are splitted squeezing very narrow transmission resonances. They arise from quasilocal states confined between $\delta$-functions. Widths of these states are determined by mixing of exponentially decaying modes with imaginary $p_{n}$ for $E+n \omega<0$; the quasilocal states are drastically narrowing when the distance between $\delta$-functions grows.

1D tight-binding model with one oscillating atom.

In this case the Eq. (5) stays valid with replacement $p_{n} \rightarrow \sqrt{\left(\Delta^{2}-(E+n \omega)^{2}\right) / \Delta}, u \rightarrow \widetilde{u} / \sqrt{\Delta}, v \rightarrow$ $\widetilde{v} / \sqrt{\Delta}$.

Figures 3a, 3c-3f present conductance relief as a function of $E_{F}$ and $v$. Sophisticated relief reflects the photonic repetitions of the permitted band boundaries (see Fig. $3 b$ ). The blockade states, situated in the $\omega$ vicin- 
ity of the permitted band boundaries, are indicated in the figures with the bold lines.

In addition to the blockade states, for $\Delta<\omega$ the reflectionless state exists. If $u=0$, this state corresponds to $E=0$. Its trace crosses the lines of blockade, forming essentially singular points with directional dependent limits. The case $u=0$ (Figs. 3a, 3e, 3f) gives symmetric pictures with respect to the permitted band center $E=0$, while the finite value of $u$ brings asymmetry (Figs. 3c, 3d).
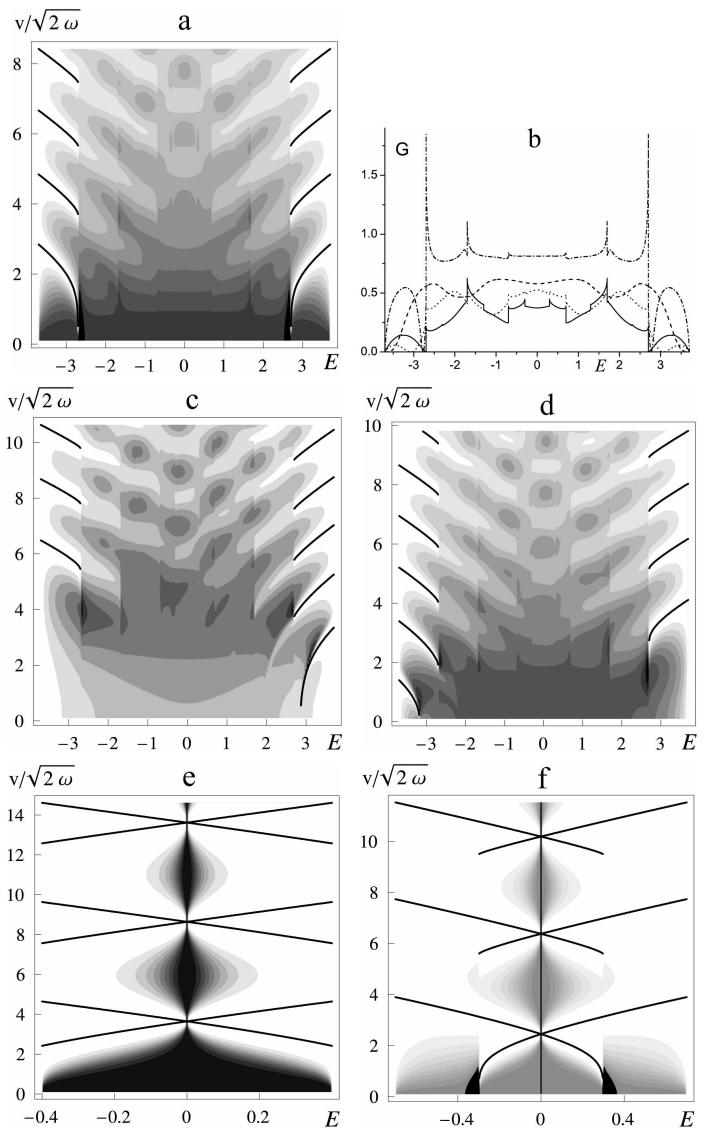

Fig. 3. (a, c-f) - Relief of conductance in the tight-binding model as a function of the Fermi energy (in units of $\omega$ ) and $v$. Figures a, c, d with $\Delta=3.7$ differ by values of $u=0$ (a), $3(\mathrm{c}),-1(\mathrm{~d})$; Figs. e and $\mathrm{f}$ with $u=0$ differ by $\Delta=0.4(\mathrm{e})$, 0.7 (f). Levels of conductance change equidistant from $<0.1$ (white) to $>1$ (black). Black lines represent the blockade states. Vertical line $E=0$ corresponds to the reflectionless state. b - Conductance versus $E_{F}$ for $\Delta=3.7, u=0$; $v=\sqrt{2}$ (dash-dotted), $v=2 \sqrt{2}$ (dashed), $v=3 \sqrt{2}$ (dots), $v=4 \sqrt{2}$ (line).
Together with the considered discrete states, this model possesses local states if $\Delta<\omega$ and $u \neq 0$. The existence of these states is conditioned by the impossibility of fulfillment of the energy conservation law for electron excitation from local state to continuum. Such states are impossible in the model of vibrating $\delta$-functions.

\section{Conclusions.}

Thus, we have demonstrated that in different models with local oscillating potential the states with zero transmission exist. This fact is unusual, because such states are impossible for static potential with limited integral. Together with these blockade states we have found the reflectionless and local states in the tightbinding model with oscillating level of a single site. The presence of blockade results in vanishing of conductance in considered systems. These systems can be used as quantum pumps. In the blockade conditions for symmetric double-well systems the derivative of photocurrent with respect to the Fermi energy also vanishes, so that the photocurrent itself has step-like behavior. So, the behavior of conductance and photocurrent looks like behavior of $\sigma_{x x}$ and $\sigma_{x y}$ in quantum Hall effect. Additionally, existence of blockade states leads in double wells to possible strong localization of states lying in the continuum.

We hope that the considered systems can be realized and utilized for confinement of electrons and fundamental measurements.

The work was supported by grant of RFBR No 05-02-16939, Program for support of scientific schools of the Russian Federation No. 4500.2006.2, the grant of the President of the Russian Federation No. MK8112.2006.2 and the Dynasty Foundation.

\section{References}

[1] D.J. Thouless, Phys. Rev. B 27, 6083 (1983).

[2] P.W. Brouwer, Phys. Rev. B 58, R10135 (1998).

[3] M. Moskalets and M. Büttiker, Phys. Rev. B 66, 205320 (2002).

[4] L.S. Braginskii, M.M. Makhmudian and M.V. Entin, Zh. Eksp. Teor. Fiz. 127, 1046 (2005) [JETP 100, 920 (2005)].

[5] M.M. Mahmoodian, L.S. Braginsky, M.V. Entin, Phys. Rev. B 74, 125317 (2006). 
[6] M.M. Mahmoodian, M.V. Entin, Europhys. Lett. 77, 67002 (2007). 